\title{
Immune gene profiling of different gut regions and gut associated lymphoid cells from rainbow trout (Oncorhynchus mykiss)
}

\author{
Sohye Yoon ${ }^{1}$, Ahmed Attaya ${ }^{1,2}$, Tiehui Wang ${ }^{1}, J_{u n} Z_{0 u}^{1}$, Chris Secombes ${ }^{1 \S}$ \\ ${ }^{1}$ Scottish Fish Immunology Research Centre, University of Aberdeen, Aberdeen, UK \\ ${ }^{2}$ Fish Diseases Laboratory, The National Institute of Oceanography and Fisheries, \\ Hurghada, Egypt
}

\begin{abstract}
Aquaculture in the UK is a valuable industry, with Scotland the largest producer of Atlantic salmon in the EU. However, infectious diseases are considered a significant factor affecting the economic stability of aquaculture, and the salmon farming industry routinely employs vaccines against viral and bacterial pathogens. Although intraperitoneal injection is very successful strategy, it has two main weaknesses: it is a costly process and there are side-effects of the adjuvants. Oral vaccination would be a method of choice but to date has not been particularly efficacious. To overcome this problem we need to have a greater understanding of the gut/mucosal immune system in fish, to allow better development of novel oral vaccination strategies for aquaculture.

Towards these goals, in this study our aim was to extend the knowledge base of fish gut immunology and to characterise immune cell markers in different gut regions for identification of the gut regions potentially responsible for antigen presentation and adaptive immune responses. From our transcriptomic analysis, it is clear that T/B cell markers and dendritic cell markers are relatively highly expressed in pyloric caeca, mid gut and hind gut, compared to oesophagus and stomach. GALT cells, isolated from pyloric caeca, mid gut and hind gut, were characterised to determine their responsiveness to a variety of stimulants, including PAMPs and recombinant cytokines. Pro-inflammatory cytokines such as IL-1 $\beta$, TNF- $\alpha$ and IL- 6 were classically upregulated post-stimulation with LPS or poly I:C but also showed a moderate increase in transcript level after PHA and flagellin (recombinant Yersinia rukeri flagellin) stimulation $24 \mathrm{~h}$ post stimulation. Th1 cell cytokines, including IFN- $\gamma$ and IL-2, were greatly induced $24 \mathrm{~h}$ post-stimulation with PHA and IL-4/13B (Th2 cytokine) was also upregulated when stimulated with PHA and poly I:C.

Overall the immune gene profiling identified here will help focus which gut regions/ cells to study following oral vaccination and we are currently verifying this with trout primed by immersion and given a commercial oral booster (AquaVac ERM Oral) vaccine.
\end{abstract}

\section{KEYWORDS}

Adaptive immunity, Antigen presenting cells, Trout, Cytokines

${ }^{\S}$ Corresponding author. Tel.: +44 (0)1224 272872

E-mail address : c.secombes@abdn.ac.uk 